\title{
Donante vivo hepático adulto-pediátrico totalmente laparoscópico. Aspectos técnicos y resultados
}

\author{
Andrés Troncoso T. ${ }^{1}$, Marcel Sanhueza G. ${ }^{1,2}$, Javier Rodríguez G. ${ }^{1}$, \\ Juan Carlos Pattilo S. ${ }^{3}$, Eduardo Briceño V. ${ }^{1}$, Juan Francisco Guerra C. ${ }^{1}$, \\ Jorge Martínez C. ${ }^{1}$, Martín Dib M. ${ }^{1}$ y Nicolás Jarufe C. ${ }^{1}$
}

'Departamento de Cirugía Digestiva. División de Cirugía. Escuela de Medicina. Pontificia Universidad Católica de Chile. Santiago, Chile. ${ }^{2}$ Cirujano digestivo. Servicio de Cirugía Hospital Dr Sótero del

Río. Santiago, Chile.

${ }^{3}$ Sección Cirugía Pediátrica.

División de Cirugía. Escuela

de Medicina. Pontificia

Universidad Católica de Chile. Santiago, Chile.

Recibido el 14 de agosto

de 2019 y aceptado para publicación el 5 de noviembre de 2019.

Correspondencia a: Dr. Andrés Troncoso T. aitt21@gmail.com
Fully laparoscopic adult living donor for pediatric transplantation. Technical aspects and results

Introduction: Laparoscopic liver surgery has presented a slower development due to the complexity it has, which determines a prolonged learning curve. In relation to laparoscopic hepatectomy for a living donor liver transplantation (LT), this has been further delayed due to concerns about donor safety, graft results and the acquisition of the technical skills of surgeons. The objective of this article is to present technical details of the laparoscopic left hepatectomy of adult living donors for pediatric transplantation and the surgical results of the cases performed in this center. Materials and Method: Presentation of the surgical technique of left hepatectomy in living donors for LT in pediatric recipients in addition to conducting a non-concurrent cohort study of patients undergoing this surgery at the Hospital Clinico Universidad Catolica between May 2011 to November 2017. Results: The series consists of 15 patients, $60 \%$ female. Thirteen patients $(86.6 \%)$ were mother or father of the recipient. $100 \%$ of patients underwent laparoscopic left hepatectomy without conversion. Clavien-Dindo Morbidity $>3$, in 1 patient who required percutaneous drainage of biloma. Median hospitalization of 3 days (2-5). There was no mortality. Conclusion: Laparoscopic left hepatectomy of living donors for pediatric LT is a safe and feasible procedure to perform in this center, with excellent results in terms of morbidity and mortality and we consider that this technique should be the route of choice for adult-pediatric living donors.

Key words: laparoscopy; hepatectomy; transplant.

\section{Resumen}

Introducción: La cirugía hepática laparoscópica ha presentado un desarrollo más lento debido a la complejidad que ésta posee, lo que determina una curva de aprendizaje prolongada. En relación a la hepatectomía laparoscópica para donante vivo en trasplante hepático (TH), ésta se ha retrasado aún más por las preocupaciones sobre la seguridad del donante, el resultado del injerto y la adquisición de las competencias técnicas de los cirujanos. El objetivo de este artículo, es exponer detalles técnicos de la hepatectomía izquierda laparoscópica de donantes vivos adultos para trasplante pediátrico y se presentan los resultados quirúrgicos de los casos realizados en este centro. Materiales y Método: Presentación de la técnica quirúrgica de la hepatectomía izquierda en donantes vivos para TH en receptores pediátricos, además, de realizar un estudio cohorte no concurrente de pacientes sometidos a esta cirugía en el Hospital Clínico de la Universidad Católica entre mayo de 2011 y noviembre de 2017. Resultados: La serie consta de 15 pacientes, $60 \%$ sexo femenino. Trece pacientes $(86,6 \%)$ eran madre o padre del receptor. El $100 \%$ de pacientes fue sometido a hepatectomía izquierda laparoscópica sin necesidad de conversión. Morbilidad Clavien-Dindo $>3,1$ paciente requirió punción percutánea de bilioma. Mediana de hospitalización de 3 días (2-5). No hubo mortalidad. Conclusión: La hepatectomía izquierda por vía laparoscópica de donante vivo para $\mathrm{TH}$ pediátrico es un procedimiento seguro y factible de realizar en este centro, con excelentes resultados en términos de morbimortalidad y consideramos que esta técnica debe ser la vía de elección para donantes vivos adultos-pediátricos.

Palabras clave: laparoscopía; hepatectomía; trasplante. 


\section{Introducción}

El trasplante hepático $(\mathrm{TH})$ es considerado el tratamiento óptimo para pacientes con enfermedad hepática terminal. El TH de donante vivo (THDV) se ha ido desarrollando progresivamente debido al aumento del número de pacientes en lista de espera para TH con un escaso número de donantes fallecidos. Por el tamaño de los receptores pediátricos, muchas veces es difícil encontrar órganos óptimos que puedan a su vez ser reducidos de manera de quedar adecuados a la necesidad de los receptores. Con la donación viva es posible elegir el tamaño y calidad de los segmentos hepáticos que se van a injertar. Al ser los donantes familiares directos de los receptores, normalmente jóvenes y en buenas condiciones de salud, la calidad de los injertos suele ser muy superior a la donación cadavérica donde, además, se agregan tiempos de isquemia más prolongados considerando la cirugía de banco que se realiza para reducción del órgano ${ }^{1}$. Los resultados quirúrgicos son al menos similares y en ocasiones mejores que con donantes fallecidos ${ }^{2,3}$. En el año 1989 se publicó el primer THDV exitoso de una madre a su hijo con atresia de vía biliar ${ }^{4}$. La primera segmentectomía lateral izquierda laparoscópica para THDV se presentó en el año $2002^{5}$. Desde entonces la técnica laparoscópica se ha desarrollado en centros altamente especializados debido a que esta cirugía no se encuentra exenta de morbilidad. Inicialmente esta técnica se introdujo de manera híbrida combinando cirugía abierta y laparoscópica ${ }^{6}$. En la actualidad, es posible realizar la totalidad de ésta se realiza por vía laparoscópica en centros altamente especializados. El objetivo de este artículo es presentar la técnica quirúrgica de la hepatectomía izquierda laparoscópica de donante vivo para receptor pediátrico y presentar los resultados quirúrgicos en términos de morbilidad de la serie de pacientes de este centro.

\section{Materiales y Método}

\section{Técnica quirúrgica}

El paciente se ubica en posición de LloydDavies $^{7}$ con el cirujano entre las piernas y ambos ayudantes a la izquierda; neumoperitoneo con $\mathrm{CO}_{2}$ y aguja de Veress. Se utiliza de rutina insuflación doble a $15 \mathrm{~mm}$ de $\mathrm{Hg}$ de manera de mantener presiones estables durante todo el procedimiento y que no declinen en casos de sangrado y/o uso de aspiración. Se introduce la óptica de $30^{\circ}$ y se explora toda la superficie hepática en busca de lesiones no pesquisadas en estudio imagenológico preoperatorio $\mathrm{y}$, posteriormente, del resto de la cavidad abdominal. Instalación de 4 trócares (supraumbilical de $10 \mathrm{~mm}$ en línea media, subcostal izquierdo de $5 \mathrm{~mm}$ en línea medio clavicular, hipocondrio derecho de $5 \mathrm{~mm}$ en línea medio clavicular e hipocondrio izquierdo de $10 \mathrm{~mm}$ en línea axilar anterior). De rutina se realiza una ecografía intraoperatoria laparoscópica de manera de pesquisar lesiones hepáticas inadvertidas y familiarizarse con la anatomía de las venas hepáticas y pedículos portales. Es muy importante considerar vena cisural o de Tanaka que en ocasiones recorre el mismo plano de la transección y, por lo tanto, aumento de los riesgos de sangrado. Posteriormente, se procede a la liberación del hígado, seccionando los ligamentos gastrohepático, coronario y triangular izquierdos (Figuras 1a, 1b, 2a y 2b); sección del ligamento de Arantius; se procede a la identificación de la vena suprahepática izquierda (Figura 3) e intentar aislarla (no siempre es posible debido a

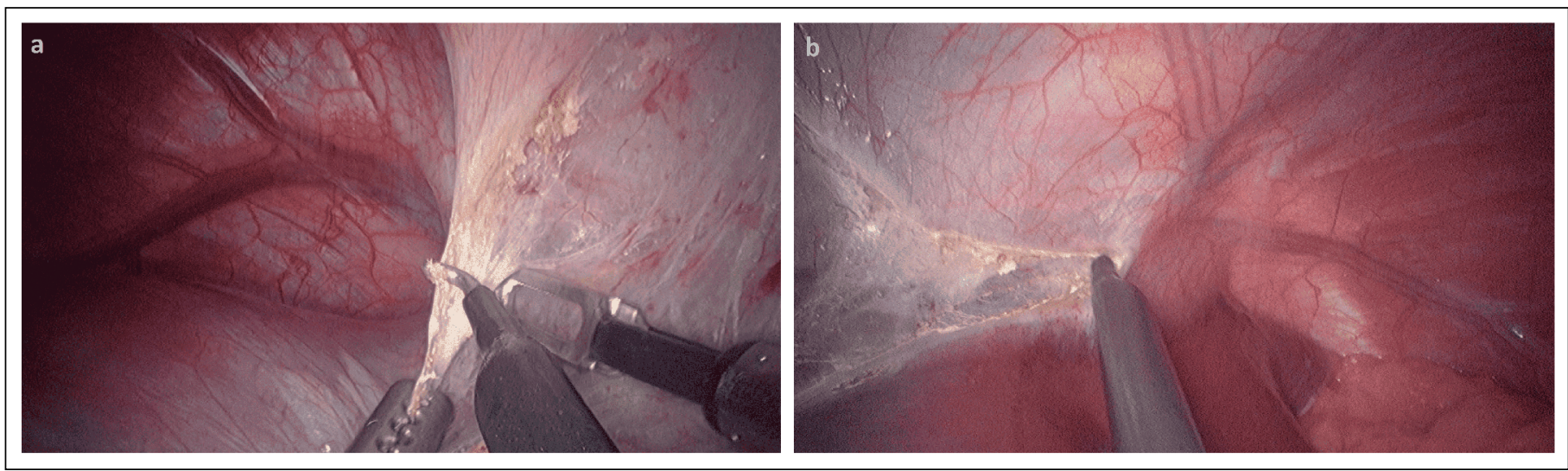

Figura 1. Liberación laparoscópica del hígado. 


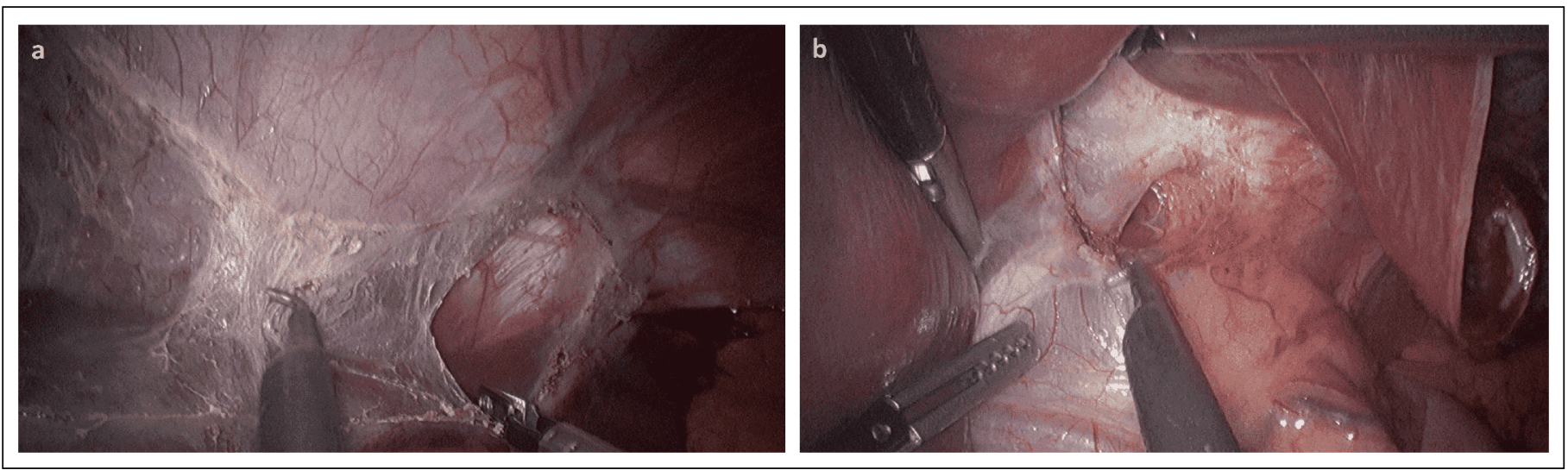

Figura 2. Liberación laparoscópica del hígado.

su emergencia conjunta con la vena suprahepática media) de lo contrario, se secciona al final de la transección del parénquima). Se realiza la disección del pedículo izquierdo aislando arteria hepática izquierda y ramas para el segmento IV (Figura 4a). Se continúa con la disección de la rama izquierda de la vena porta (Figura 4a). En ocasiones es necesario seccionar una rama al segmento caudado (Figura 4b) de manera de permitir mayor libertad y longitud de la porta izquierda para ser aislada y separada de la bifurcación portal. En este punto el flujo arterial y portal deben ser preservados, incluso durante toda la transección del parénquima. Se procede, posteriormente, a la sección del puente de parénquima hepático entre los segmentos 3 y 4 cuando está presente; demarcación de línea de transección de parénquima hepático (Figura 5a). Es importante destacar que la transección del parénquima comienza en el segmento $4 \mathrm{~b}$, alejado unos 2-3 cm a la derecha del ligamento redondo de manera de obtener un conducto biliar izquierdo antes de su bifurcación en los segmentos 2 y 3 y, por ende, más expedito para el implante. Posteriormente, se realiza la transección del parénquima con bisturí armónico y bipolar de manera combinada, ligando con clips

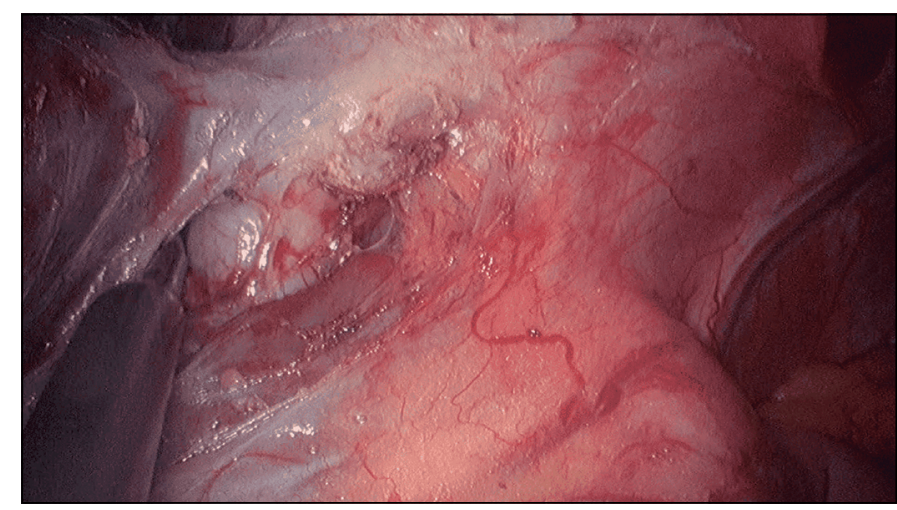

Figura 3. Vena suprahepática izquierda.

estructuras vasculares de mayor tamaño que deben ser identificadas durante la transección (Figura 5b). El conducto biliar izquierdo sólo se secciona una vez avanzado en la transección del parénquima (Figura 6) y previa realización de colangiografía intraoperatoria transcística de manera de evitar daño biliar

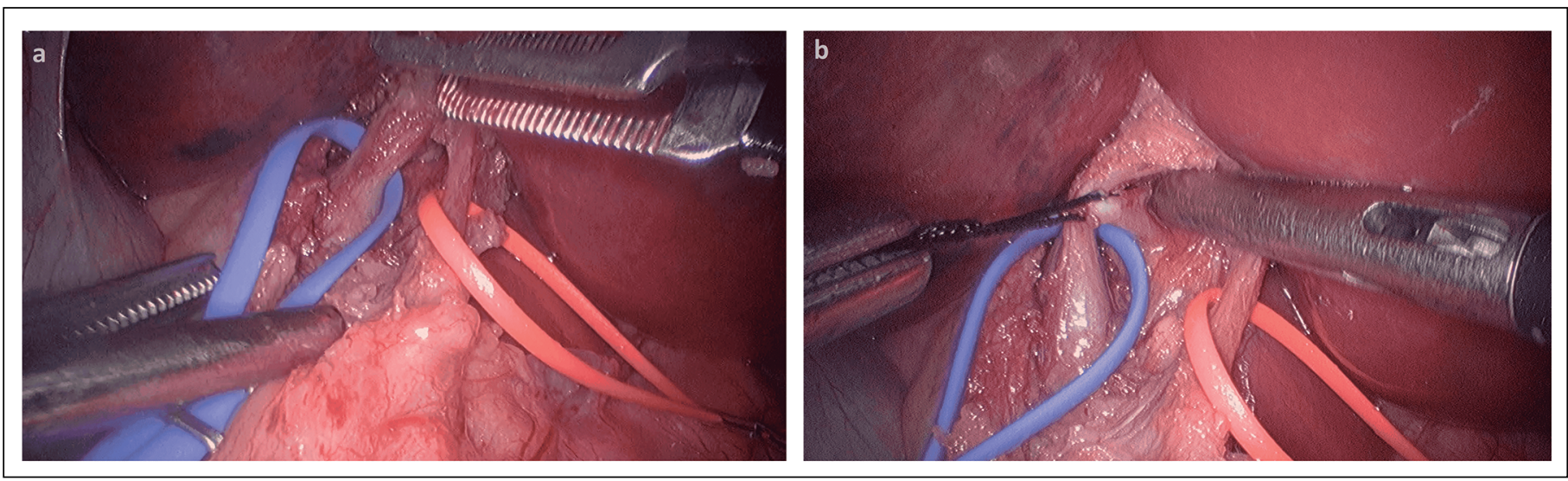

Figura 4. Disección del pedículo hepático izquierdo. 
contralateral. Posterior a la colangiografía, se realiza además en esta etapa, la colecistectomía. Previo a la sección del pedículo hepático se realiza una laparotomía de Pfannenstiel (transversa suprapúbica) para la extracción de la pieza operatoria, a fin de minimizar el tiempo de isquemia. Finalmente, las estructuras del pedículo hepático se seccionan (ligadura de arteria hepática con Hem-o-lok ${ }^{\circledR}$ y clips; ligadura con doble Hem-o-lok ${ }^{\circledR}$ a vena porta izquierda (Figuras 7 y 8), seguidas,, posteriormente, por la vena suprahepática izquierda la cual se secciona con un stapler vascular (Figura 9). Habitualmente, se deja un drenaje de tipo aspirativo al lecho extrayéndose por uno de los sitios de inserción de los trócares.
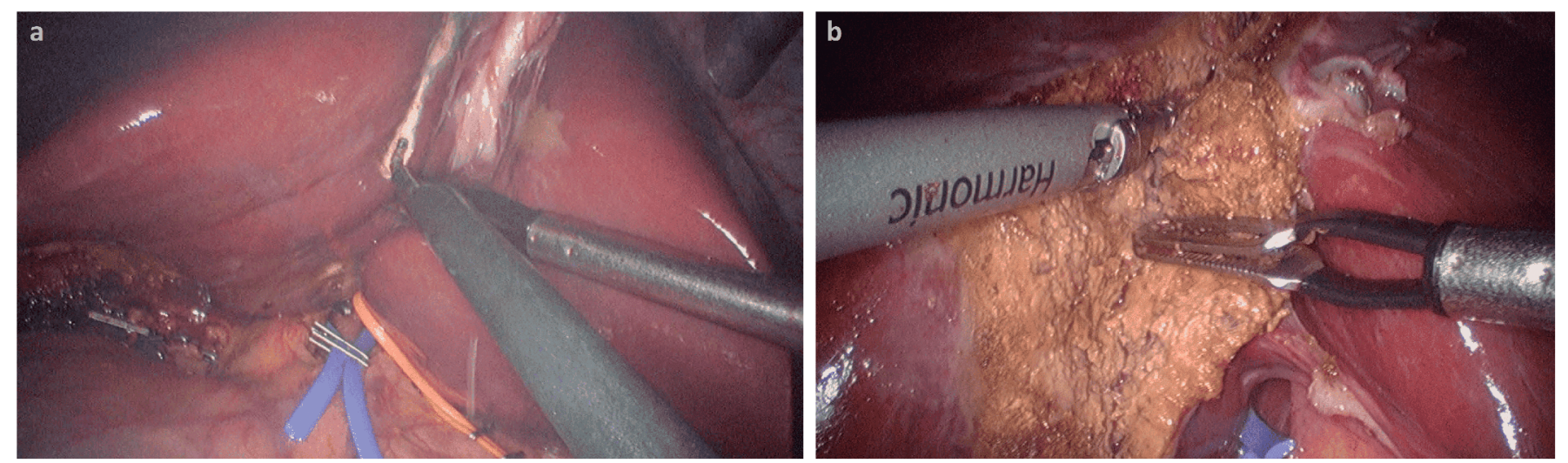

Figura 5. Transección de parénquima hepático.

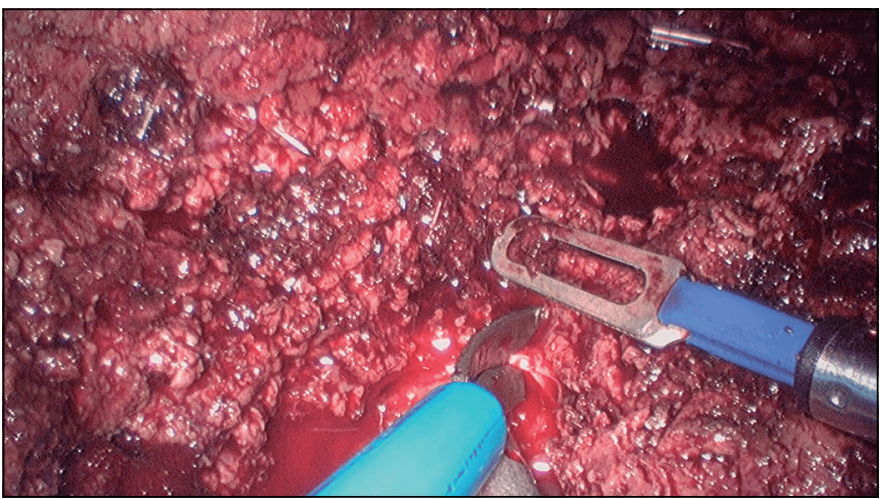

Figura 6. Sección de conducto biliar.

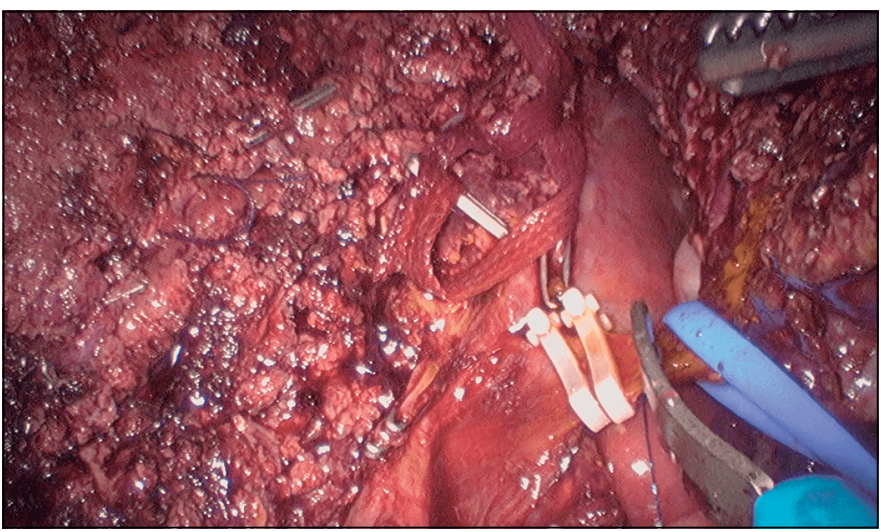

Figura 8. Control vascular intraparenquimatoso.

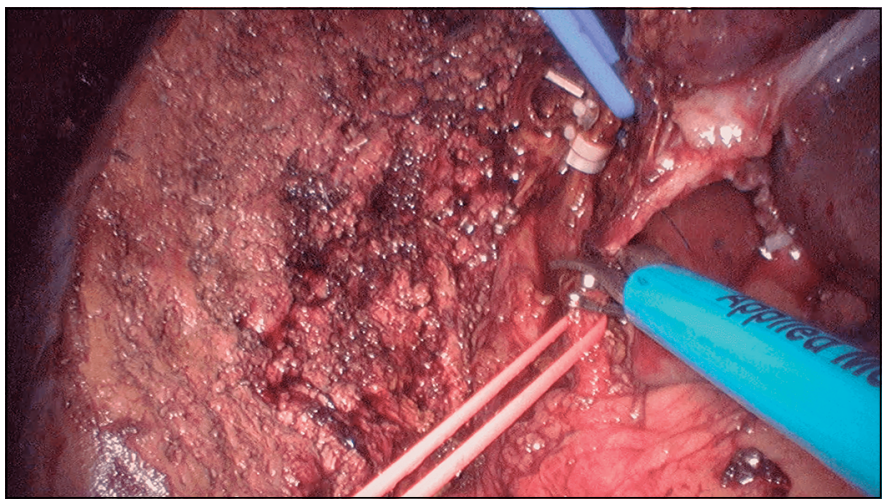

Figura 7. Control vascular intraparenquimatoso.

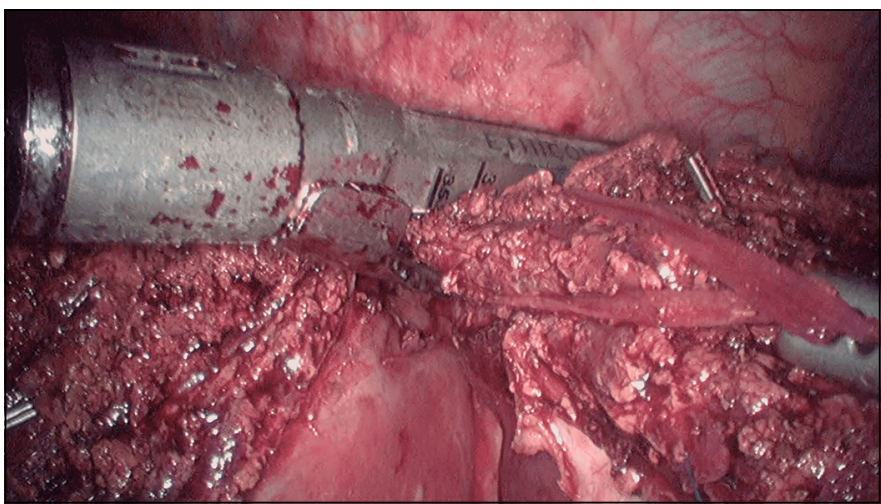

Figura 9. Sección vena suprahepática izquierda con sutura mecánica. 


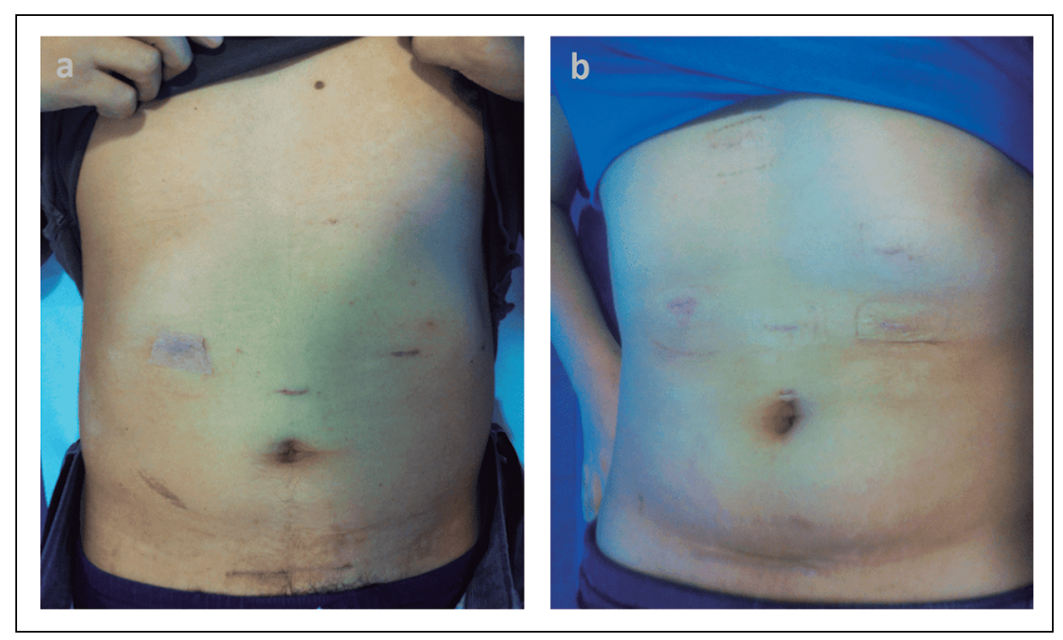

Figura 10. Cicatrices postoperatorias.

\section{Metodología}

Se realizó un estudio de cohorte no concurrente de pacientes que fueron seleccionados y sometidos a hepatectomía izquierda laparoscópica de donante vivo adulto para $\mathrm{TH}$ en receptor pediátrico desde mayo de 2011 hasta noviembre de 2017 en el Hospital Clínico de la Pontificia Universidad Católica de Chile (HCPUC). Se incluyeron todos los pacientes evaluados por equipo multidisciplinario del HCPUC del período señalado y en quienes, finalmente, se realizó la hepatectomía izquierda laparoscópica. Respecto de la maniobra, se realizó estudio imagenológico preoperatorio para volumetría hepática y evaluación de anatomía biliar, arterial y portal con resonancia nuclear magnética (RNM) y estudio de laboratorio con hemograma, pruebas de función hepática, función renal, grupo sanguíneo ( $\mathrm{ABO}$ - $\mathrm{Rh})$ y pruebas de coagulación en todos los pacientes. Una vez realizados los estudios se evalúa potencial donante en reunión multidisciplinaria de equipo de programa de $\mathrm{TH}$ con psiquiatra, hepatólogo, radiólogo y cirujano de trasplante. Se analizaron variables biodemográficas, clínicas, de laboratorio y de morbimortalidad. Base de datos diseñada en programa Microsoft Excel $^{\circledR}$ v.16.11.1 y análisis estadístico en programa SPSS $^{\circledR}$ v.22. Morbilidad según Clavien-Dindo ${ }^{7}$.

\section{Resultados}

La serie consta de 15 pacientes, 9 de sexo femenino $(60 \%)$ y 6 de sexo masculino $(40 \%)$. Edad promedio de 35,3 (25-62) años. Sólo 1 paciente con co- morbilidad que correspondió a un hipotiroidismo en tratamiento y control óptimo. Ningún paciente con antecedente de cirugía abdominal previa. Respecto del parentesco, 13 pacientes $(86,6 \%)$ eran padre o madre del receptor y en 2 receptores $(13,4 \%)$, los donantes fueron su tío y abuela respectivamente. Índice de masa corporal promedio de $24,8(17,7-28,4)$ $\mathrm{kg} / \mathrm{m}^{2}$. El $100 \%$ de pacientes con perfil hepático en exámenes de laboratorio dentro de rangos normales.

Todos los pacientes fueron sometidos a hepatectomía izquierda laparoscópica con la técnica previamente descrita, sin necesidad de conversión a cirugía abierta. Doce pacientes $(80 \%)$ clasificación tipo I de la American Society of Anesthesiologists (ASA) y 3 pacientes ASA II (20\%). Tiempo quirúrgico promedio de $196 \pm 54 \mathrm{~min}$. No se presentaron complicaciones en el intraoperatorio ni se requirieron transfusiones de hemoderivados.

Morbilidad según Clavien-Dindo en 5 pacientes $(33,3 \%)$, sólo uno grado III (drenaje percutáneo de bilioma). Dos pacientes presentaron una fístula biliar de bajo débito que fue tratada de manera conservadora difiriendo el retiro del drenaje, sin requerir tratamiento quirúrgico, endoscópico o percutáneo; otros 2 pacientes presentaron biliomas, uno de los cuales requirió punción percutánea y un paciente presentó un hematoma de lecho operatorio tratado de manera conservadora.

La mediana de hospitalización fue de 3 días (25). No hubo mortalidad intrahospitalaria ni a los 90 días postcirugía.

\section{Discusión}

El objetivo de la hepatectomía laparoscópica de donante vivo es obtener la mejor calidad del injerto con la máxima seguridad para el donante otorgándole los beneficios de la cirugía mini-invasiva ${ }^{5}$. Además, en nuestro país de baja tasa de donación, permite disminuir la lista de pacientes pediátricos en espera de TH. A través de los resultados ya publicados y de esta serie de pacientes, se ha mostrado la factibilidad y seguridad de realizar este procedimiento con una baja morbilidad.

La introducción de esta técnica ha sido lenta debido principalmente a la curva de aprendizaje de la hepatectomía por vía laparoscópica, siendo una de las más importantes preocupaciones el control del sangrado 9,10 .

La posición anterior del lóbulo hepático izquierdo es una condición anatómica favorable para realizar la hepatectomía izquierda por vía laparoscópica para donante vivo, además, que posee limitadas 
variaciones anatómicas ${ }^{11}$. Sin embargo, del punto de vista de la técnica quirúrgica uno de los principales problemas es la sección del conducto biliar izquierdo a un nivel adecuado, es por esto que el estudio imagenológico preoperatorio es fundamental, además, de la confirmación intraoperatoria a través de colangiografía transcística que se realizó de rutina en esta serie.

Los estudios de Soubrane (2006) $)^{12}$ Kim $(2011)^{13}$ y Scatton $(2015)^{1}$, corresponden a las 3 series de pacientes más importantes que se refieren a esta técnica. Respecto del punto de vista del donante, la vía laparoscópica se asoció a menor pérdida sanguínea, mejor evolución postoperatoria con disminución de la tasa de complicaciones y estadía hospitalaria más corta comparada a la vía abierta.

El riesgo de mortalidad en los donantes vivos es la principal preocupación. De acuerdo con los resultados del European Liver Transplant Registry, con un registro de 3.622 procedimientos realizados en 74 centros entre octubre de 1991 y diciembre de 2009 , la mortalidad de los donantes fue de un $0,16 \%$ con una tasa de morbilidad global de un $20 \%$. En esta serie de pacientes presentamos morbilidad en 5 pacientes $(33 \%)$ sin mortalidad.

Existe una prolongada curva de aprendizaje para la cirugía hepática mini-invasiva, probablemente una de las principales razones por las cuales esta técnica no se ha difundido globalmente. Hoy en día están en desarrollo promisorios modelos de simulación y cirugía en animales que permitirían reducir estas curvas. En los centros donde se realizan intervenciones laparoscópicas hepato-bilio-pancreáticas de rutina junto con cirugía de trasplante, es posible asociar ambas técnicas para lograr realizar cirugías de donante hepático por laparoscopía con seguridad razonable tanto para el donante como el receptor.

La hepatectomía izquierda por vía laparoscópica de donante vivo para $\mathrm{TH}$ pediátrico es un procedimiento seguro y factible de realizar en este centro, permitiendo a los donantes tener todas las ventajas de la cirugía laparoscópica en relación a la cirugía abierta, con excelentes resultados en términos de morbimortalidad y consideramos que esta técnica debe ser la vía de elección para donantes vivos adultos-pediátricos.

\section{Responsabilidades éticas}

Protección de personas y animales. Los autores declaran que para esta investigación no se han realizado experimentos en seres humanos ni en animales.

Confidencialidad de los datos. Los autores declaran que en este artículo no aparecen datos de pacientes.

Conflictos de interés: no hay.

\section{Bibliografía}

1. Scatton O, Katsanos G, Boillot O, Goumard C, Bernard D, Stenard F, et al. Pure laparoscopic left lateral sectionectomy in living donors: From innovation to development in France Ann Surg. 2015;261:506-12.

2. Bourdeaux C, Darwish A, Jamart J, Tri TT, Janssen M, Lerut J, et al. Livingrelated versus deceased donor pediatric liver transplantation: A multivariate analysis of technical and immunological complications in 235 recipients. Am J Transplant. 2007;7:440-7.

3. Gurevich M, Guy-Viterbo V, Janssen M, Sthephenne X, Smets F, Sokal E, et al. Living Donor Liver Transplantation in Children: Surgical and Immunological Results in 250 Recipients at Université Catholique de Louvain. Ann Surg. 2015;262:1141-9.
4. Nagasue N, Kohno H, Matsuo S, Yamanoi A, Uchida M, Takemoto Y, et al. Segmental (partial) liver transplantation from a living donor. Transplant Proc. 1992;24:1958-9.

5. Cherqui D, Soubrane O, Husson E, Barshashz E, Vignaux O, Ghimouz M, et al. Laparoscopic living donor hepatectomy for liver transplantation in children. Lancet 2002;359(9304):392-6.

6. Koffron AJ, Kung R, Baker T, Fryer J, Clark L, Abecassis M. Laparoscopicassisted right lobe donor hepatectomy. Am J Transplant. 2006;6:2522-5.

7. Dindo D, Demartines N, Clavien PA. Classification of surgical complications: a new proposal with evaluation in a cohort of 6336 patients and results of survey. Ann Surg. 2004;240:205-13.

8. Jarufe N, Marambio A, De La Llera JF, Varas J, Sanhueza M, Martínez J. Rev Chil Cir. 2013;65:463-71.
9. Kasahara M, Sakamoto S, Fukuda A. Pediatric living-donor liver transplantation. Semin Pediatr Surg. 2017;26:224-32.

10. Park JI, Kim KH, Lee SG. Laparoscopic living donor hepatectomy: A review of current status. J Hepatobiliary Pancreat Sci. 2015;22:779-88.

11. Cherqui D. Laparoscopic liver resection. Br J Surg. 2003;90:644-6.

12. Soubrane O, Cherqui D, Scatton O, Stenard F, Bernard D, Branchereau S, et al. Laparoscopic left lateral sectionectomy in living donors: Safety and reproducibility of the technique in a single center. Ann Surg. 2006;244:815-20.

13. Kim KH, Jung DH, Park KM, Lee YJ, Kim DY, Kim KM, et al. Comparison of open and laparoscopic live donor left lateral sectionectomy. Br J Surg. 2011;98:1302-8. 